\title{
AERONAUTICAL QUARTERLY
}

A Journal devoted to Aeronautics and the Allied Sciences Volume $X X$ May 1969

Part 2

\section{CONTENTS}

The Calculations of Three-Dimensional Turbulent Boundary Layers; (iii) Comparison of Attachment-Line Calculations with Experiment and M. R. Head

Application of the Discrete Element Method to the Buckling Analysis of Rectangular Plates Under Arbitrary Membrane Loading

D. J. Dawe

Displacement Effects on Pitot Tubes with Rectangular Mouths

A. Quarmby and H.K. Das

An Experimental study of the Flexure of Some Triangular Sections

B. Nath

Correlation of Constant Pressure Turbulent Boundary Layer Measurements on Insulated Walls

J. A. P. Stoddart

Vibrations of Tapered Cantilever Beams and Shafts

Vibration Characteristics of Straight Blades of Asymmetrical Aerofoil Cross-Section

A. V. Krishna Murty K. R. Prabhakaran

W. Carnegie and $B$. Dawson

An Improved Reversed-Flow Formulation of the Galerkin-KantorovichDorodnitsyn Multi-Moment Integral Method

H.E. Bethel

Errata

LONDON

ROYAL AERONAUTICAL SOCIETY 4 HAMILTON PLACE, W1V OBQ 


\title{
THE AERONAUTICAL QUARTERLY
}

\author{
Editorial Board
}

Dr. A. M. BALlantyne, OBE. TD, BSc, PhD, FRAeS. HonfCaSi, FAiAa (Chairman)

Sir Robert CoCkburn, KBE, CB. PhD, MSc, Fraes

Sir WILliam FARREN, CB. MBE, MA, DSc, FRS, Honfraes. HonfaiAA

Sir ARnold Hall, MA, frs, Honfraes, HonfaiaA

Professor D. W. Holder, MA, FRS, FRAeS

Lord KINGS NORTON, DSc, DIC, PhD, FRAeS, FAIAA

Sir Alfred Pugsley, Obe, DSc, Frs, Honfraes

Dr. P. B. WALKER, CBE, MA. PhD. FRAeS

Editor-Miss E. C. PtKe, MA, Afraes

\section{Editorial Referees}

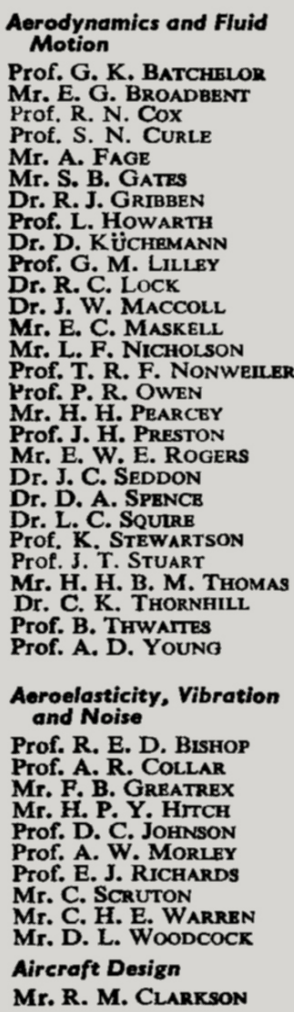

Dr. H. H. GARDNER

Mr. G. H. LEE

Mr. R. L. LICKLEY

Sir MORIEN MORGAN

Dr. A. E. RuSSELL

Dr. A. E. RUSSELL
Dr. R. S. STAFFORD

\section{Air Transport}

Mr. A. C. Campbell ORde

Mr. P. G. MASEFIFLD

Mr. B. S. SHENSTONE

Mr. W. TYE

\section{Astronautics and Guided} Flight

Mr. J. E. Allen

Mr. A. D. BAXTER

Mr. E. G. C. BURT

Mr. A. v. CleAver

Mr. D. J. FARRAR

Mr. G. C. I. GARDINER

Mr. D. J. LYONS

Mr. W. H. STEPHENS

\section{Aviation and Space
Medicine}

Dr. K. G. Berorn

Dr. A. BUCHANAN BARBour

Dr. W. E. Hick

Control and Guidance
Mr. J. F. ATHERTON
Mr. B. G. GATES
Dr. F. E. JONES
Mr. W. MAKINSON
Dr. R. L. SMTTH ROSE
Dr. A. M. UTTLEY
Instruments and Equipment
Dr. G. E. BAIRSTO
Mr. A. E. BINGHAM
Mr. W. J. CHARNLEY
Mr. R. GRAHAM

Mr. C. D. Holland

Dr. C. S. Hudson

Mr. C. N. JAQUES

Mr. H. TEMPI.ETON

Materials and Metallurgy

Prof. L. Arrchison

Dr. N. P. AlLeN

Dr. W. M. DOYLE

Dr. G. FORREST

Sir Nevill MotT

Dr. B. P. Muluins

Mr. L. ROTHERHAM

Dr. H. Surton

Dr. C. SYKeS
Mathematics and Numerical Analysis

Dr. E. T. GoODWIN

Dr. S. H. Hollunadale

Prof. G. TEMPLB

Meteorology and Space Environment

Mr. P. J. MEADE

Prof. R. S. SCORER

Prof. P. A. SHEPTARD

\section{Performance and Flight} Testing

Mr. H. DAvies

Mr. R. R. DUDDY

Mr. J. HANSON

Mr. P. A. Hufton

Mr. D. R. NeWMAN

Mr. J. N. OUICK

Propulsion

Mr. E. L. Bass

Mr. S. L. BRAGG

Mr. H. G. ConwaY

Dr. J. W. DRINK WaTER

Sir A. H. ROY FEDDEN

Prof. W. E. GARNRE

Dr. S. G. HOOKER

Prof. J. H. HORLOCK
Mr. A. R. Howr.l

Mr. W. H. LINDSEY

Mr. P. LLOYD

Dr. E. S. MOULT

Mr. F. NixON

Mr. F. M. OWNER

Mr. H. Pearson

Sir OWEN SAUNDERS

Prof. A. G. SMITH

Dr. B. S. STRATFORD

Rotorcraft and Air Cushion Vehicles

Prof. J. A. J. BenNext

Mr. R. HAFNER

Dr. G. S. HisLoP

Prof. W. A. MAIR

Mr. R. STANTON JONES

Structures and Solid Body

Mechanics

Prof. D. N. DE G. AlleN

Dr. I. M. AlLison

Prof. J. H. AROYRIS

Mr. R. J. AtKInson

Dr. A. J. BARRETT

Prof. W. CarNegie

Prof. A. H. CHILVER

Mr. H. L. Cox

Prof. W. S. Hemp

Prof. R. HILL

Prof. H. G. Hopkins

Mr. H. B. HOWARD

Mr. E. D. KeEN

Dr. D. M. A. LegGeTt

Dr. E. H. MANSFIELD

Mr. L. S. D. MORLEY

Mr. M. J. PARKS

Prof. S. C. ReDShaw

Mr. R. H. SANDIFER

Mr.R. L. WHEELER

Prof. W. H. WIrTRICK

VTOL/STOL

Prof. D. KerTH-Lucas

Dr. R. C. PANKHURS

Dr. JOHN WRLAM 